\title{
HIV and the kidney in the acute medical unit
}

\author{
Authors: John W Booth ${ }^{A}$ and Frank A Post ${ }^{B}$
}

Acute kidney injury (AKI) is encountered commonly in HIVpositive patients admitted to the acute medical unit. The spectrum of AKI has changed in the era of combination anti-retroviral therapy, and now includes adverse effects of commonly used anti-retroviral drugs in addition to traditional precipitants such as severe sepsis or exposure to nephrotoxic antimicrobials. An accurate diagnosis requires careful integration of clinical data including volume status, history of potentially nephrotoxic exposures and consideration of immuno-virological status. This article provides an overview of common causes of AKI in HIV and presents a framework by which the acute care physician may approach the finding of an elevated serum creatinine in a patient with HIV.

\section{Introduction}

Acute kidney injury (AKI) remains a common problem in HIV-positive individuals. Many risk factors for AKI are shared with the general population, including pre-existent chronic kidney disease (CKD), hypertension, diabetes and use of angiotensin-converting-enzyme inhibitors (ACE-I) or angiotensin receptor blockers (ARB), each serving to lower the threshold for renal injury in the context of hypovolaemia or sepsis. ${ }^{1}$ In addition, HIV-infected patients often have unique risk factors for AKI, either associated with immunodeficiency, immune reconstitution or nephrotoxic effects of antiretroviral therapy (ART). ${ }^{2-4}$ It is common for AKI to reflect the multifactorial effects of these renal 'stressors', and careful evaluation of the patient's pre-morbid condition, immunovirological status, current clinical condition and recent nephrotoxic exposures is required for an accurate diagnosis and to individualise treatment. The 'STOP' (sepsis, toxins, obstruction, parenchymal disease) mnemonic remains a useful aide memoire for considering contributing factors of AKI in $\mathrm{HIV}$, as per the general population.

A number of 'intrinsic' renal diseases, most notably HIVassociated nephropathy (HIVAN), ${ }^{5}$ may present as AKI in HIV (Table 1). The presence of blood and protein on urine dipstick may point to glomerulonephritis (eg HIV-associated

Authors: A specialty registrar, UCL Centre for Nephrology, Royal Free Hospital NHS Foundation Trust, London, UK; ${ }^{B}$ reader in HIV medicine, King's College Hospital NHS Foundation Trust, London, UK immune complex glomerulonephritis, post-infectious glomerulonephritis or bacterial endocarditis), rhabdomyolysis or thrombotic microangiopathy (TMA), although urinalysis is a poor predictor of the renal 'compartment of injury' (ie glomerular vs tubulo-interstitial injury) in HIV. ${ }^{6}$ Traditional pointers to tubulo-interstitial nephritis (TIN), such as fever, rash and peripheral eosinophilia, are equally insensitive. ${ }^{6}$ Hence, renal biopsy represents an important definitive diagnostic tool in unexplained or non-resolving AKI.

AKI should be staged using the 'kidney disease: improving global outcomes' (KDIGO) criteria (Table 2) and early nephrology input sought in cases of progressive renal impairment or AKI stage III. Fluid resuscitation to correct any prevailing volume deficit is of paramount importance, and should follow recently published guidance from NICE. ${ }^{8}$ Renally excreted drugs, such as zidovudine, lamivudine, tenofovir and emtricitabine, require dose adjustment in renal impairment. Indications for renal replacement therapy are the same as those for the general population.

Beside AKI, renal presentations encountered in HIV include nephrotic syndrome (eg HIVAN, immune complex glomerulonephritis), chronic kidney disease (Table 3) and disorders of fluid and electrolytes (eg Fanconi syndrome due to tenofovir). While these syndromes are discussed in brief where relevant, the main focus of this article is AKI.

\section{Drugs and AKI}

Medications, in particular anti-microbial drugs, frequently cause or contribute to AKI in HIV (Table 1). Common mechanisms of injury include acute tubular injury, TIN or crystalluria with sludging and tubular obstruction. AKI should be pre-empted when drugs prone to crystallising in urine (eg aciclovir, sulphadiazine) are prescribed, by careful dose adjustment for reductions in glomerular filtration rate (GFR) and maintenance of good hydration and urinary flow rate. Drug withdrawal is sufficient to reverse AKI in most scenarios, although corticosteroid treatment may be warranted in severe cases of TIN.

ACE-I or ARB use is now common among HIV-positive patients. While these medications can exert long-term nephroprotective effects through lowering of glomerular filtration pressure, this property impairs renal autoregulation in the face of circulatory stress, and may compound AKI and hyperkalaemia. ACE-I and ARB should be stopped immediately in patients presenting with AKI, and suspended pre-emptively in dehydration or sepsis. Non-steroidal anti-inflammatory 


\section{Table 1. The spectrum of AKI encountered in patients with HIV.}

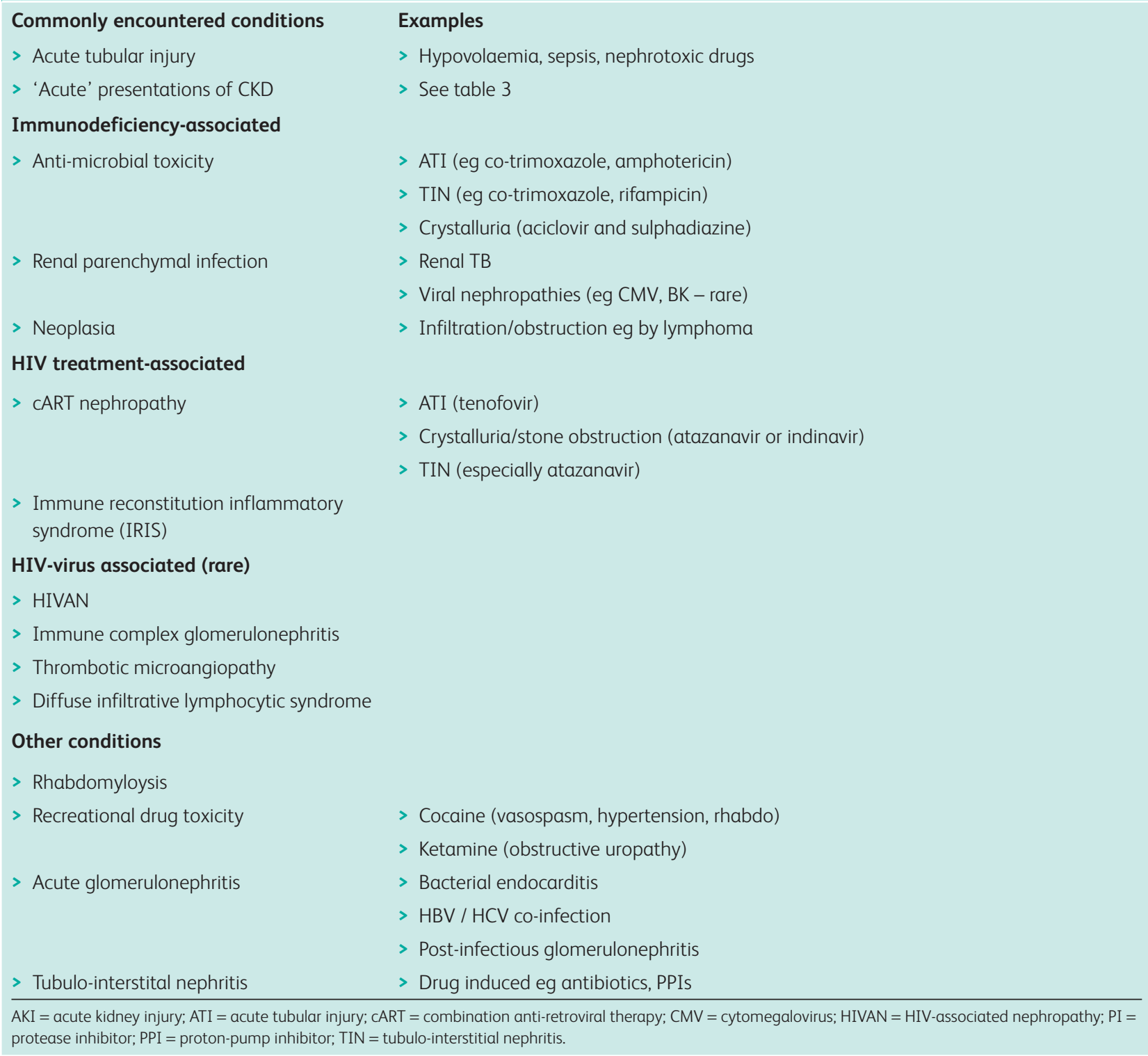

drugs (NSAIDs) are also commonly implicated in AKI and should be stopped; their use should be avoided in those with pre-existing CKD. Many episodes of severe AKI may be avoided through patient education to suspend ACE-I, ARB or NSAIDs during periods of intercurrent illness until medical advice has been sought.

Several anti-retrovirals (dolutegravir, rilpivirine, ritonavir and cobicistat) may increase serum creatinine concentrations by inhibiting renal tubular transporters and others (tenofovir, atazanavir and lopinavir) have been associated with the development of CKD. ${ }^{9,10}$ Two drugs, tenofovir and atazanavir, may cause kidney injury which should be considered in patients presenting with AKI. ${ }^{11}$

\section{Tenofovir}

Tenofovir disoproxil fumarate (TDF), a widely used nucleotide reverse transcriptase inhibitor may cause severe proximal renal tubulopathy (Fanconi syndrome) characterised by hypophosphataemia, tubular (low molecular weight) proteinuria, glycosuria, hypokalaemia and metabolic acidosis. ${ }^{12}$ Hypophosphataemia may be severe and present acutely with

\section{Table 2. KDIGO staging of AKI.}

$\begin{array}{ll}\text { Stage } & \text { Serum creatinine } \\ 1 & 1.5-1.9 \text { times baseline OR } \\ & \geq 26.5 \mu \mathrm{mol} / \mathrm{L} \text { increase } \\ 2 & 2.0-2.9 \text { times baseline } \\ 3 & \geq 3.0 \text { times baseline OR } \\ & \geq 354 \mu \mathrm{mol} / \mathrm{L} \text { OR initiation of } \\ & \text { RRT }\end{array}$

\section{Urine output}

$<0.5 \mathrm{~mL} / \mathrm{kg} / \mathrm{h}$ for $6-12 \mathrm{~h}$

$<0.5 \mathrm{~mL} / \mathrm{kg} / \mathrm{h}$ for $\geq 12 \mathrm{~h}$

$<0.3 \mathrm{~mL} / \mathrm{kg} / \mathrm{h}$ for $\geq 24 \mathrm{~h}$ OR anuria for $\geq 12 \mathrm{~h}$

AKI = acute kidney injury; RRT = renal replacement therapy. Adapted with permission. ${ }^{7}$ 


\section{Table 3. Spectrum of CKD in patients with HIV infection.}

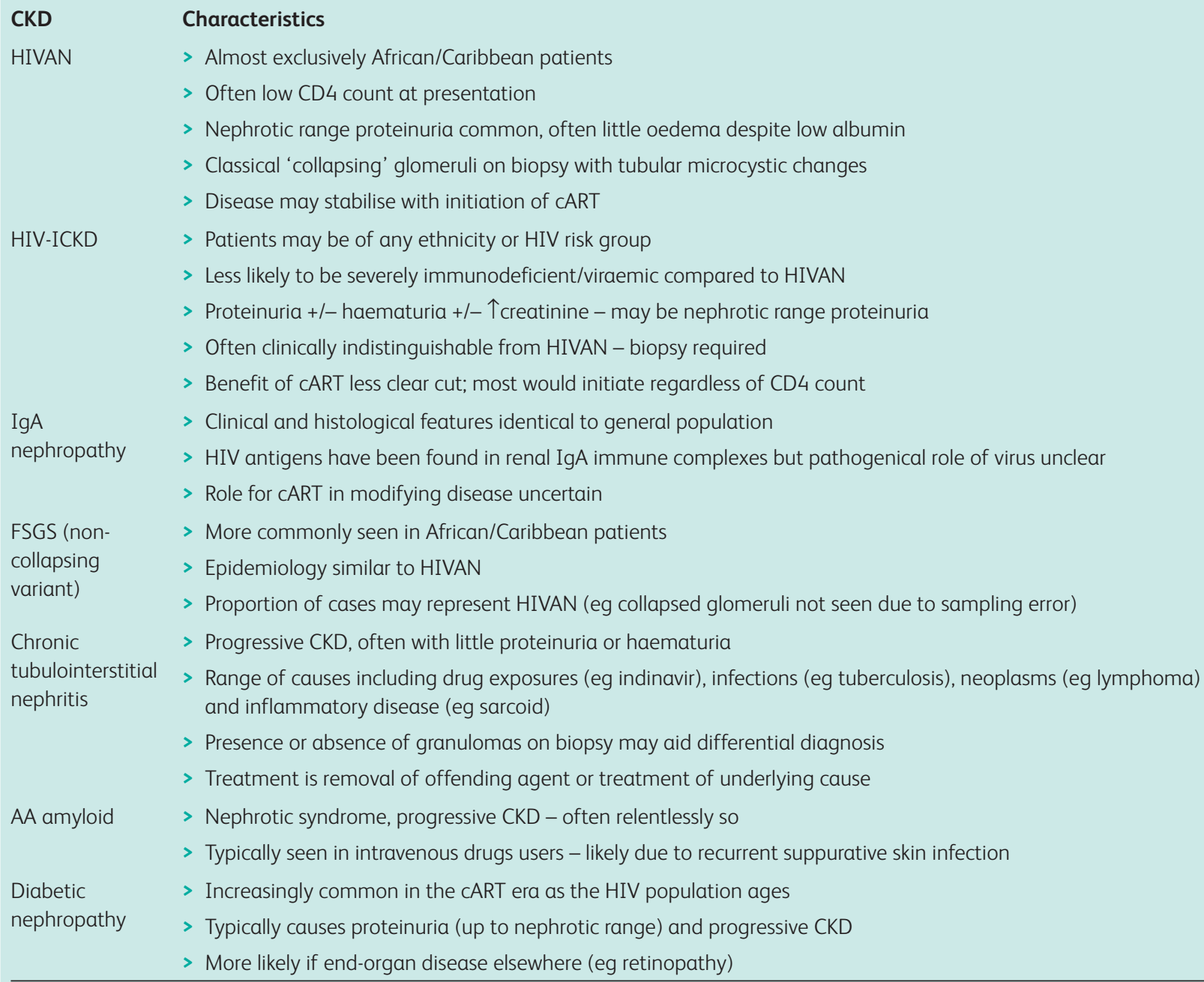

CART = combination antiretroviral therapy; CKD = chronic kidney disease; HIVAN = HIV-associated nephropathy; HIV-ICKD = HIV immune-complex kidney disease; MCGN = mesangio-capillary glomerulonephritis; NOS = not otherwise specified; VL = viral load.

\section{Key points}

Remember important causes of AKI in HIV with 'STOP' mnemonic.

Assessment of fluid status and early fluid resuscitation is paramount.

Seek, treat and monitor for complications of AKI (eg hyperkalaemia).

Adjust drug doses (including antiretrovirals) for renal function.

Tenofovir and atazanavir have renal adverse effects which may present as AKI.

Involve the multidisciplinary team early (HIV team, renal team, pharmacist).

KEYWORDS: HIV, AKI, ART, tenofovir, atazanavir bone pain or pathological fracture due to osteomalacia. ${ }^{13}$ Risk is highest in older patients, those with prior CKD and those who take tenofovir with ritonavir-boosted protease inhibitors (PIs). ${ }^{12}$ Elevated creatinine may be a late feature.

Patients taking TDF are also at risk of developing acute tubular injury without features of Fanconi syndrome, sometimes triggered by sepsis or volume depletion. Kidney biopsy in such cases typically shows enlarged distorted mitochondria within proximal tubular cells, frequently visible as eosinophilic inclusions on light microscopy. ${ }^{14}$ Tenofovir should be substituted in stage II or III AKI and advice sought from an HIV physician regarding appropriate alternatives. A new formulation of tenofovir (tenofovir alafenamide) with an improved renal safety profile is currently in development. ${ }^{15}$

\section{Atazanavir}

Atazanavir is a PI frequently used in combination with ritonavir (as a 'boosting' agent). Like indinavir, an obsolete 
Fig 1. Approach to the HIV-infected patient presenting with an elevated serum creatinine. *See Table 2 for KDIGO staging of AKI. ACE-I = angiotensin converting enzyme inhibitor; $A K I$ = acute kidney injury; $A N A=$ anti-nuclear antibody; $A N C A=$ anti-neutrophic cytoplasmic antibody; $A R B=$ angiotensin receptor blocker; $\mathrm{CK}=$ creatine kinase; $C K D=$ chronic kidney disease; eGFR = estimated glomerular filtration rate; $\mathrm{HBV}=$ hepatitis $B$ virus; $\mathrm{HCV}=$ hepatitis $C$ virus; IRIS = immune reconstitution inflammatory syndrome; $\mathrm{LDH}=$ lactate dehydrogenase; NRTI = nucleoside reverse transcriptase inhibitor; $\mathrm{RhF}=$ rheumatoid factor; $R R T$ = renal replacement therapy; SBE = subacute bacterial endocarditis; TMA = thrombotic microangiopathy.

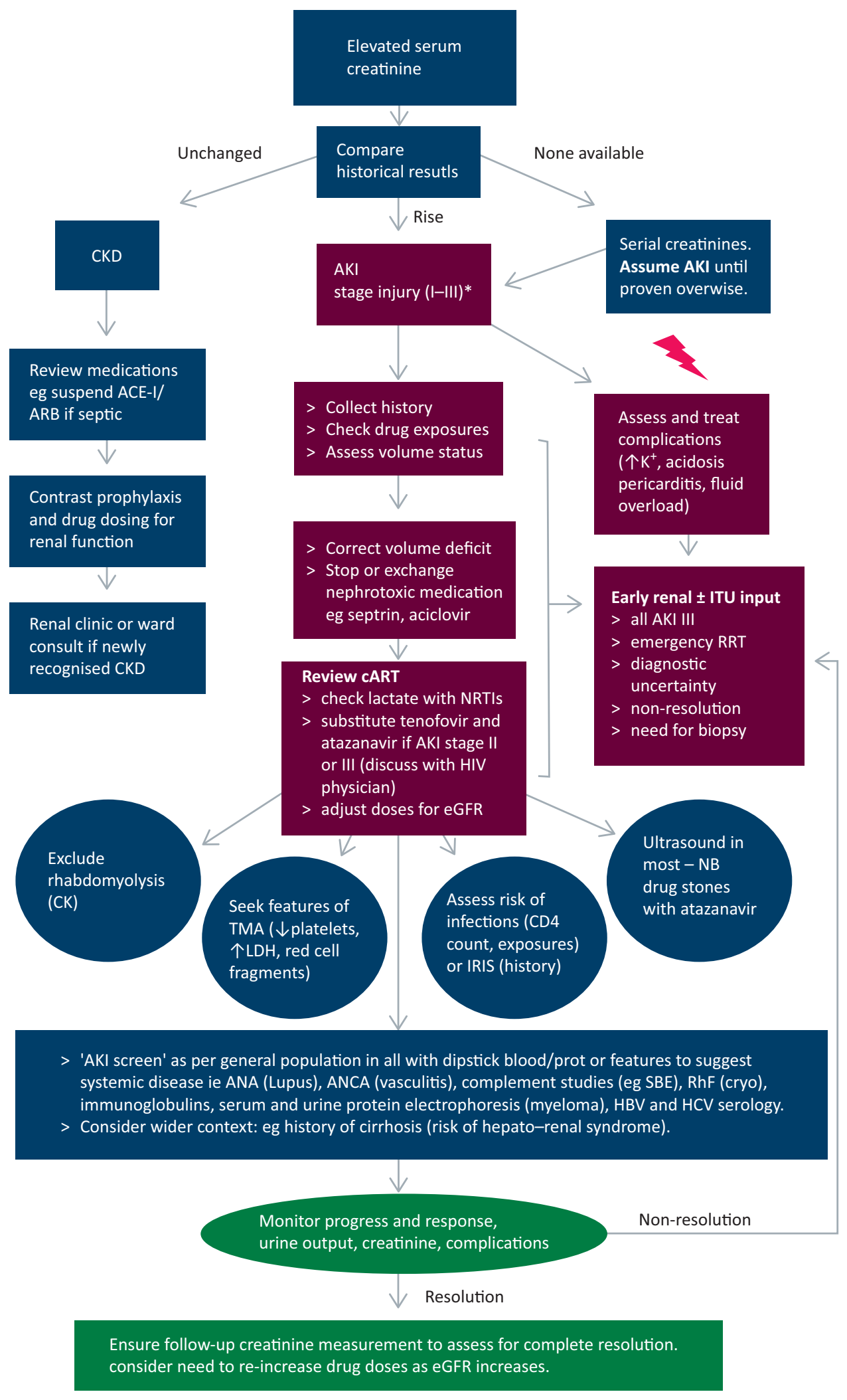


first-generation PI, atazanavir may be associated with renal tract crystallisation and radiolucent calculi, albeit at lower frequency. ${ }^{16}$ Although rare, atazanavir has also been associated with acute TIN, and a more insidious, scarring chronic granulomatous TIN driven by intra-tubular crystal deposition. ${ }^{17}$ Atazanavir is best avoided in the setting of AKI.

\section{Infection, malignancy and liver disease}

While ischaemic acute tubular injury commonly complicates severe opportunistic infection, direct renal injury by infective agents is also relatively common. Tuberculosis may present with sub-acute progressive renal impairment, with or without the classic 'sterile' pyuria and granulomatous interstitial inflammation on biopsy. Syphilis remains common among men who have sex with men and may induce an abrupt onset nephrotic syndrome of membranous type. Viral infections, including cytomegalovirus and adenovirus, may rarely cause renal tubulo-interstitial inflammation in the severely immunocompromised host, and viral inclusion bodies may be visible on histology.

Lymphoma remains common in HIV-positive patients and may be associated with renal impairment or AKI either due to direct lymphomatous infiltration of the kidney, ureteric compression or indirect effects including nephrotoxic chemotherapy and tumour lysis syndrome. Chronic liver disease, for instance due to co-infection with hepatitis B virus (HBV) or $\mathrm{C}(\mathrm{HCV})$, is a common comorbid condition among patients presenting with AKI, and hepato-renal syndrome may need to be considered where AKI is unresponsive to fluid resuscitation. Co-infection should also prompt consideration of viral glomerulonephritis (eg membranous nephropathy with $\mathrm{HBV}$ ) or cryoglobulinaemia (HCV).

\section{'Acute' presentations of CKD}

The spectrum of disorders presenting as CKD in HIV differs considerably from those presenting as AKI (Table 3). It is not uncommon for HIVAN to present as AKI or rapidly progressive renal impairment over weeks to months, and for HIVAN to be the first manifestation of previously unrecognised HIV infection. ${ }^{5}$ Nephrotic-range proteinuria, a common feature of HIVAN, also lowers the threshold for acute tubular injury during intercurrent illness. In the absence of previous creatinine measurements for comparison, serial blood tests are essential to determine if renal function is stable or deteriorating acutely. While reduced renal size on imaging signifies chronicity, the inverse does not hold true; renal size is often normal or increased in chronic disorders such as HIVAN where renal volume is expanded by tubular microcystic dilatation.

\section{Immune disorders and thrombotic microangiopathy}

Immune reconstitution inflammatory syndrome (IRIS), triggered by the recent introduction of ART with consequent rise in CD4 count, may present as AKI. Renal IRIS usually manifests as granulomatous interstitial nephritis with a $\mathrm{CD} 4^{+}$rich lymphocytic infiltrate, most commonly directed against previously unrecognised mycobacterial infection. ${ }^{18}$ ART can be continued in the majority of cases, with addition of oral corticosteroids and relevant anti-microbial therapy (eg anti- tuberculous chemotherapy).

Diffuse infiltrative lymphocytosis syndrome (DILS) is uncommon since the advent of widespread ART, but may present with progressive renal impairment as a consequence of tubulo-interstitial renal infiltration by oligoclonal $\mathrm{CD} 8{ }^{+} \mathrm{T}$ cells. DILS presents classically as a Sjogren-like syndrome with parotid enlargement and sicca symptoms due to lymphocyte infiltration. ${ }^{19}$ Initiation of ART is the mainstay of treatment, although corticosteroids do appear to have an adjunctive role in the presence of significant renal disease.

TMA, manifesting either as thrombotic thrombocytopaenic purpura or haemolytic uraemic syndrome, is associated with severe immunodeficiency and is now encountered rarely. The association of AKI with thrombocytopaenia in HIV should trigger evaluation for other features of TMA such as red cell fragments on blood film, elevated serum lactate dehydrogenase or depressed haptoglobins. Treatment includes plasma exchange, prompt initiation of ART (preferably as a oncedaily regimen and dosed post-plasmapheresis) and supportive therapy including haemodialysis where necessary. ${ }^{20}$

\section{Approach to the HIV patient with AKI}

A framework by which to approach AKI encountered in a patient with HIV is presented in Fig 1. Review of historical renal function tests will provide immediate insight into whether an elevated serum creatinine represents AKI or CKD. Life-threatening complications of AKI (including hyperkalaemia and severe acidosis) should be sought early and treatment initiated as appropriate. Dehydration should be corrected with intravenous fluid therapy, and nephrotoxic medication (including NSAIDs, ACE-I or ARB) suspended. ART should be reviewed, doses adjusted for GFR, and tenofovir and atazanavir suspended or substituted in stage II or III AKI. Further history, examination and investigations should be tailored toward the differential diagnosis of AKI presented in Table 1.

Response to treatment should be monitored by tracking urine output and serial creatinine measurements. Severe AKI (including all stage III), diagnostic uncertainty or nonresolution should trigger nephrology consultation. Criteria for renal replacement therapy are identical to AKI in the general population.

\section{Conclusion}

While the incidence of AKI in HIV-positive patients has declined with widespread use of ART, those with immunodeficiency and comorbidities remain at increased risk of AKI. Tenofovir and atazanavir may infrequently be a cause of AKI and are best avoided in patients with AKI. The management of AKI is supportive, with appropriate fluid resuscitation, avoidance of nephrotoxic medications and treatment of underlying diseases.

\section{Disclosures}

JWB has received conference funding from Shire Pharmaceuticals. FAP has received funding to attend conferences or educational meetings, honoraria and/or research grants from Gilead Sciences, Bristol-Myers Squibb, Janssen-Cilag, GlaxoSmithKline/ViiV healthcare and Merck. 


\section{References}

1 Roe J, Campbell LJ, Ibrahim F, Hendry BM, Post F. HIV care and the incidence of acute renal failure. Clin Infect Dis 2008;47:242-9.

2 Li Y, Shlipak MG, Grunfeld C, Choi AI. Incidence and risk factors for acute kidney injury in HIV Infection. Am J Nephrol 2012;35: $327-34$.

3 Herlitz LC, Mohan S, Stokes MB et al. Tenofovir nephrotoxicity: acute tubular necrosis with distinctive clinical, pathological and mitchondrial abnormalitis. Kidney Int 2010;78:1171-7.

4 Ibrahim F, Naftalin C, Cheserem E et al. Immunodeficiency and renal impairment are risk factors for HIV-associated acute renal failure. AIDS 2010;24:2239-44.

5 Post FA, Campbell LJ, Hamzah L et al. Predictors of renal outcome in HIV-associated nephropathy. Clin Infect Dis 2008;46:1282-9.

6 Parkhie SM, Fine DM, Lucas GM, Atta MG. Characteristics of patients with HIV and biopsy-proven acute interstitial nephritis. Clin J Am Soc Nephrol 2010;5:798-804.

7 KDIGO. Clinical practice guideline for acute kidney injury. Brussels: KDIGO, 2012. Available online at http://www.kdigo.org/ clinical_practice_guidelines/pdf/KDIGO\%20AKI\%20Guideline.pdf [Accessed 5 October 2015].

8 National Institute for Health and Care Excellence. Intravenous fluid therapy in adults in hospital. CG174. London: NICE, 2013.

9 Ryom L, Mocroft A, Kirk O et al. Association between antiretroviral exposure and renal impairment among HIV-positive persons with normal baseline renal function: the D:A:D study. J Infect Dis 2013;207:1359-69.

10 Mocroft A, Kirk O, Reiss P et al. Estimated glomerular filtration rate, chronic kidney disease and antiretroviral drug use in HIVpositive patients. AIDS 2010;24:1667-78.

11 Yombi JC, Pozniak A, Boffito M et al. Antiretrovirals and the kidney in current clinical practice: renal pharmacokinetics, alterations of renal function and renal toxicity. AIDS 2014;28:621-32.
12 Hall AM, Hendry BM, Nitsch D, Connolly JO. Tenofovir-associated kidney toxicity in HIV-infected patients: a review of the evidence. Am J Kidney Dis 2011;57:773-80.

13 Woodward CL, Hall AM, Williams IG et al. Tenofovir-associated renal and bone toxicity. HIV Med 2009;10:482-7.

14 Hamzah L, Booth JW, Jose S et al. Renal tubular disease in the era of combination anti-retroviral therapy. AIDS 2015;14:1831-6.

15 Sax PE, Wohl D, Yin M et al. Tenofovir alafenamide versus tenofovir disoproxil fumarate, coformulated with elvitegravir, cobicistat, and emtricitabine, for initial treatment of HIV-1 infection: two randomised, double-blind, phase 3, non-inferiority trials. Lancet 2015;385:2606-15.

16 Rockwood N, Mandalia S, Bower M, Gazzard B, Nelson M. Ritonavir-boosted atazanavir exposure is associated with an increased rate of renal stones compared with efavirenz, ritonavir-boosted lopinavir and ritonavir-boosted darunavir. AIDS 2011;25:1671-3.

17 Hara M, Suganuma A, Yanagisawa N et al. Atazanavir nephrotoxicity. Clin Kidney J 2015;8:137-42.

18 Lawn SD, Bekker LG, Miller RF. Immune reconstitution disease associated with mycobacterial infections in HIVinfected individuals receiving antiretrovirals. Lancet Infect Dis 2005;5:361-73.

19 Kazi S, Cohen PR, Williams F, Schempp R, Reveille JD. The diffuse infiltrative lymphocytosis syndrome. Clinical and immunogenetic features in 35 patients. AIDS 1996;10:385-91.

20 Hart D, Sayer R, Miller R et al. Human immunodeficiency virus associated thrombotic thrombocytopenic purpura--favourable outcome with plasma exchange and prompt initiation of highly active antiretroviral therapy. Br J Haematol 2011;153:515-9.

Address for correspondence: Dr JW Booth, UCL Centre for Nephrology, UCL Medical School, Rowland Hill Street, London NW3 2PF, UK.

Email: johnwrbooth@doctors.org.uk

\title{
Isolated microscopic haematuria of glomerular origin: clinical significance and diagnosis in the 21st century
}

\author{
Authors: Melanie MY Chan ${ }^{A}$ and Daniel P Gale ${ }^{B}$
}

Isolated microscopic, or non-visible, haematuria of glomerular origin was previously regarded a benign finding, but it is now known that, even in the absence of proteinuria, hypertension or renal impairment at presentation, haematuria is associated with increased risk of kidney failure in the long term. The most common causes of isolated microscopic haematuria among

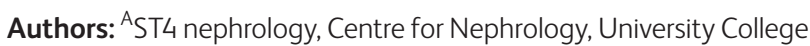
London, Royal Free Hospital, London, UK; ${ }^{\text {B }}$ senior clinical research fellow and honorary consultant, Centre for Nephrology, University College London, Royal Free Hospital, London, UK children and young adults are IgA nephropathy, Alport syndrome (AS), and thin basement membrane nephropathy (TBMN). AS, which is usually inherited as an X-linked or autosomal recessive trait, and TBMN, which is usually autosomal dominant, are caused by mutations in the genes encoding type-IV collagen, an abundant component of the glomerular basement membrane. A detailed family history with screening of at-risk relatives is important, allowing prompt diagnosis of affected relatives and helping determine the mode of transmission. As costs fall and availability increases, genetic testing is increasingly being used in clinical practice to provide diagnostic and predictive information for patients and their families. 